\title{
Hyperglycemia increases the risk of death in extremely preterm baboons
}

\author{
Cynthia L. Blanco', Lisa L. McGill-Vargas', Donald McCurnin' and Amy R. Quinn'
}

BACKGROUND: Transient neonatal hyperglycemia (HG) has been reported in up to $80 \%$ of extremely preterm human infants. We hypothesize that severe $\mathrm{HG}$ is associated with increased morbidity and mortality in preterm baboons.

METHODS: Sixty-six baboons born at $67 \%$ of gestation were studied. HG was defined as serum glucose level $\geq 150 \mathrm{mg} / \mathrm{dl}$ during the first week of life. Animals were stratified into two groups: severe $\mathrm{HG}$ ( $\geq 8$ events) and nonsevere $\mathrm{HG}$ ( $<8$ events).

RESULTS: $\mathrm{HG}$ developed in 65 of the 66 (98\%) baboons that were included. A total of 3,386 glucose measurements were obtained. The mean serum glucose level was $159 \pm 69 \mathrm{mg} / \mathrm{dl}$ for the severe HG group and $130 \pm 48 \mathrm{mg} / \mathrm{dl}$ for the nonsevere HG group during the first week of life. No differences were found in gender, birth weight, sepsis, patent ductus arteriosus, or oxygenation/ventilation indexes between groups. Severe HG was associated with early death even after controlling for sepsis, postnatal steroid exposure, and catecholamine utilization.

CONCLUSION: $\mathrm{HG}$ is common in preterm baboons and is not associated with short-term morbidity. Severe HG occurring in the first week of life is associated with early death in preterm baboons.

$\mathbf{T}$ ransient neonatal hyperglycemia (HG) has been reported in up to $80 \%$ of extremely preterm infants and has been associated with increased morbidity, including intraventricular hemorrhage, white matter injury, retinopathy of prematurity, and necrotizing enterocolitis (1-7). Furthermore, both early and persistent HG during the first week of life are associated with higher mortality in extremely preterm infants $(2,6,8)$. The pathogenesis seems to be multifactorial and is probably secondary to defective islet $\beta$-cell processing/secretion of insulin, decreased peripheral insulin sensitivity, and hepatic insulin resistance $(5,9,10)$.

$\mathrm{HG}$ is highly prevalent in the preterm baboon and has a reported incidence similar to that in extremely preterm human infants $(3,11)$. Baboons have $97 \%$ phylogenetic proximity with humans, and when born preterm, they develop conditions unique to preterm infants, such as respiratory distress syndrome, patent ductus arteriosus, and bronchopulmonary dysplasia $(12,13)$.

Baboons have been shown to be a pertinent nonhuman primate model for examining the underlying cellular/molecular mechanisms responsible for insulin resistance, which can develop spontaneously along with diabetes in adult baboons
$(14,15)$. Furthermore, preterm fetal baboons have significant downregulation of insulin-signaling proteins such as AS160, GLUT1, and GLUT4 in the skeletal muscle, which may be implicated in the development of HG in preterm infants (11).

The preterm baboon is the only animal that survives extreme prematurity ( $>48 \mathrm{~h}$ ) in which HG develops spontaneously. Another advantage of the model is the homogeneity of the animals included in this study, due to the strict protocols for each major organ system and their narrow gestational age. In addition, common perinatal events that lead to prematurity are not present (i.e., intrapartum infection and/or maternal disease) and therefore confounding factors are minimized. The objective of this study was to evaluate whether severe $\mathrm{HG}$ is associated with increased morbidity and mortality in preterm baboons.

\section{RESULTS}

Of the 66 preterm baboons included in the study, 65 (98\%) had at least one episode of $H G$ within the first $3 \mathrm{~d}$ of life; all had at least one episode by $7 \mathrm{~d}$ of age. A total of $91 \%$ of animals had more than one episode of HG during the first week of life. Nineteen of the study animals were classified as having severe HG according to the criteria described in the Methods section. No differences in gender or birth weight were found between the severe and nonsevere HG groups (Table 1). As expected, $100 \%$ of all study animals were exposed prenatally to steroids. The demographic details are summarized in Table 1.

The development of sepsis was similar between the severe and nonsevere HG groups (Table 1). The amount of catecholamines utilized during the first 3,7 , or all days of life, in order to maintain a normal mean arterial pressure, was similar between the two groups (Table 1). In addition, the maximum amount and the mean amount of catecholamines per day were calculated for each day that the baboons were alive and were not associated with the development of HG (not significant). Serum glucose levels did not correlate with the amount of catecholamine infused $\left(r^{2}=0.001\right)$. Twenty $(30 \%)$ of the study animals were exposed to postnatal steroids (hydrocortisone), with no differences between the two stratified groups (Table 1).

\section{Glucose Levels and Nutritional Data}

A total of 3,386 glucose measurements were obtained throughout the study period (Figure 1). For the first week of age, the 
Table 1. Demographic characteristics

\begin{tabular}{|c|c|c|c|}
\hline Study characteristic & $\begin{array}{c}\text { Nonsevere } \\
\text { hyperglycemia } \\
(n=47)\end{array}$ & $\begin{array}{c}\text { Severe } \\
\text { hyperglycemia } \\
(n=19)\end{array}$ & $P$ value \\
\hline Birth weight (g) & $373 \pm 41$ & $364 \pm 45$ & 0.458 \\
\hline Male & $30(63 \%)$ & $9(47 \%)$ & 0.21 \\
\hline Prenatal steroids & $100 \%$ & $100 \%$ & 1.0 \\
\hline Patent ductus arteriosus & $100 \%$ & $100 \%$ & 1.0 \\
\hline Sepsis & $2(4.3 \%)$ & $1(5.6 \%)$ & 0.83 \\
\hline Length of stay (d) & $13 \pm 2.5$ & $11 \pm 5.2$ & 0.81 \\
\hline Early death* & $5(10 \%)$ & $6(32 \%)$ & 0.03 \\
\hline Postnatal steroids & $13(28 \%)$ & $7(37 \%)$ & 0.46 \\
\hline \multicolumn{4}{|l|}{ Hyperglycemic episodes ( $n$ ) } \\
\hline First $3 d^{*}$ & $4.8 \pm 3.2$ & $8.5 \pm 2.9$ & $<0.001$ \\
\hline First $7 d^{*}$ & $6.6 \pm 3.0$ & $11.5 \pm 3.3$ & $<0.001$ \\
\hline Glucose (mean) first $3 \mathrm{~d}^{*}(\mathrm{mg} / \mathrm{dl})$ & $135 \pm 57$ & $170 \pm 74$ & $<0.001$ \\
\hline Glucose (mean) first $7 \mathrm{~d}^{*}(\mathrm{mg} / \mathrm{dl})$ & $130 \pm 48$ & $159 \pm 69$ & $<0.001$ \\
\hline Glucose (max) first $3 \mathrm{~d}^{*}(\mathrm{mg} / \mathrm{dl})$ & $226 \pm 58$ & $291 \pm 77$ & $<0.001$ \\
\hline Glucose (max) first $7 \mathrm{~d}^{*}(\mathrm{mg} / \mathrm{dl})$ & $243 \pm 57$ & $295 \pm 76$ & $<0.01$ \\
\hline $\begin{array}{l}\text { Glucose (max-min) first } 3 d^{*} \\
(m g / d l)\end{array}$ & $166 \pm 69$ & $241 \pm 83$ & $<0.001$ \\
\hline $\begin{array}{l}\text { Glucose (max-min) first } 7 d^{*} \\
(m g / d l)\end{array}$ & $187 \pm 68$ & $249 \pm 81$ & $<0.01$ \\
\hline GIR first $3 \mathrm{~d}$ (mg/kg/min) & $5.3 \pm 1.0$ & $5.4 \pm 1.3$ & 0.18 \\
\hline GIR first 7 days (mg/kg/min) & $6.3 \pm 1.6$ & $6.3 \pm 2.0$ & 0.98 \\
\hline Insulin doses* & $2.1 \pm 2.7$ & $4.5 \pm 2.8$ & 0.002 \\
\hline Catecholamines all days ${ }^{\mathrm{a}}(\mathrm{ml})$ & $0.13 \pm 0.15$ & $0.14 \pm 0.19$ & 0.80 \\
\hline Catecholamines first $3 \mathrm{~d}^{\mathrm{a}}(\mathrm{ml})$ & $0.23 \pm 0.22$ & $0.18 \pm 0.23$ & 0.43 \\
\hline Catecholamines first $7 \mathrm{~d}^{\mathrm{a}}(\mathrm{ml})$ & $0.20 \pm 0.20$ & $0.16 \pm 0.21$ & 0.47 \\
\hline $\begin{array}{l}\text { Oxygenation index } \\
\text { first } 3 \mathrm{~d}\end{array}$ & $7.3 \pm 3.5$ & $6.8 \pm 1.6$ & 0.46 \\
\hline $\begin{array}{l}\text { Oxygenation index } \\
\text { first } 7 \mathrm{~d}\end{array}$ & $6.7 \pm 2.8$ & $6.1 \pm 1.5$ & 0.41 \\
\hline Ventilation index first $3 d$ & $52.8 \pm 20.2$ & $51.1 \pm 7.9$ & 0.64 \\
\hline Ventilation index first $7 \mathrm{~d}$ & $49.9 \pm 17.2$ & $47.5 \pm 8.2$ & 0.46 \\
\hline Total feeding volume (cc) & $95.8 \pm 55.9$ & $85.5 \pm 65.1$ & 0.25 \\
\hline $\begin{array}{l}\text { Average feeding volume (cc/ } \\
\mathrm{kg} / \mathrm{d} \text { ) }\end{array}$ & $18.8 \pm 11.2$ & $17.0 \pm 13.2$ & 0.26 \\
\hline Sodium first $3 \mathrm{~d}$ (meq/dl) & $146 \pm 8$ & $147 \pm 10$ & 0.11 \\
\hline Sodium first $7 \mathrm{~d}$ (meq/dl) & $146 \pm 8$ & $146 \pm 10$ & 0.21 \\
\hline $\begin{array}{l}\text { Potassium first } 3 \mathrm{~d} \\
(\mathrm{meq} / \mathrm{dl})^{*}\end{array}$ & $3.4 \pm 0.6$ & $3.3 \pm 0.6$ & $<0.001$ \\
\hline $\begin{array}{l}\text { Potassium first } 7 \mathrm{~d} \\
\text { (meq/dl) }\end{array}$ & $3.6 \pm 0.6$ & $3.6 \pm 0.7$ & 0.90 \\
\hline Hematocrit first $3 \mathrm{~d}(\mathrm{meq} / \mathrm{dl})^{*}$ & $45.2 \pm 8.1$ & $44.0 \pm 7.4$ & $<0.01$ \\
\hline Hematocrit first $7 \mathrm{~d}(\mathrm{meq} / \mathrm{dl})$ & $40.9 \pm 8.1$ & $40.5 \pm 7.2$ & 0.16 \\
\hline
\end{tabular}

Data are shown as mean $\pm S D$, unless otherwise specified.

GIR, glucose infusion rate; max, maximum; meq, milliequivalents.

aCatecholamines are calculated from the cumulative $\mathrm{ml}$ of dopamine, dobutamine, and epinephrine given hourly during all days alive, the first $3 \mathrm{~d}$, or the first $7 \mathrm{~d}$ for all animals. All catecholamines are mixed with the same concentration per body weight. ${ }^{*} P<0.05$ by $\chi^{2}$-Tests and $t$-tests for categorical and continuous data, respectively.

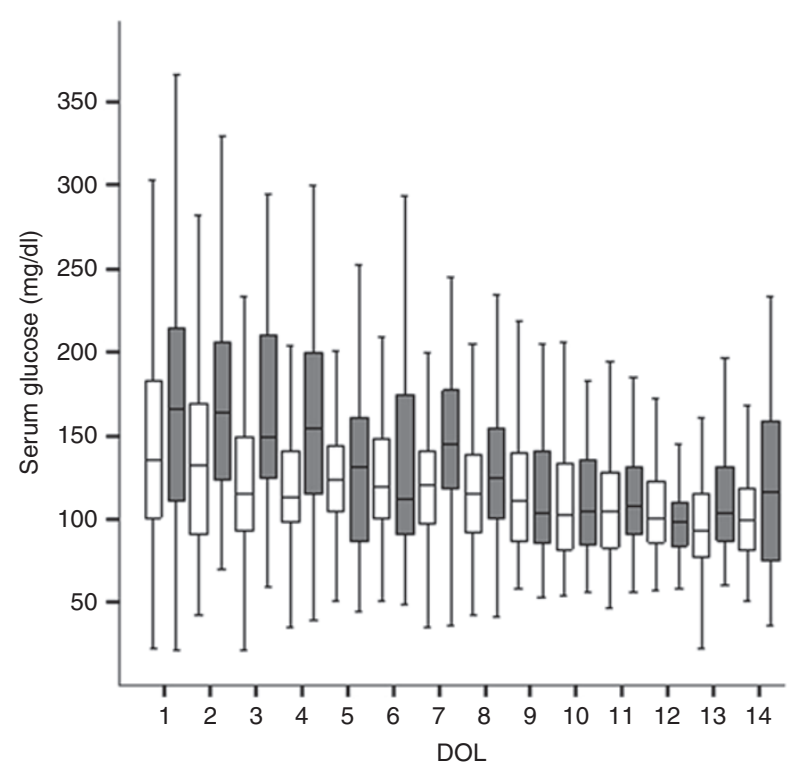

Figure 1. Daily serum glucose. Daily serum glucose measurements are represented as medians in the figure, with interquartile ranges shown as black lines. The nonsevere HG group is shown in white boxes and the severe HG group in dark boxes. DOL, day of life; HG, hyperglycemia.

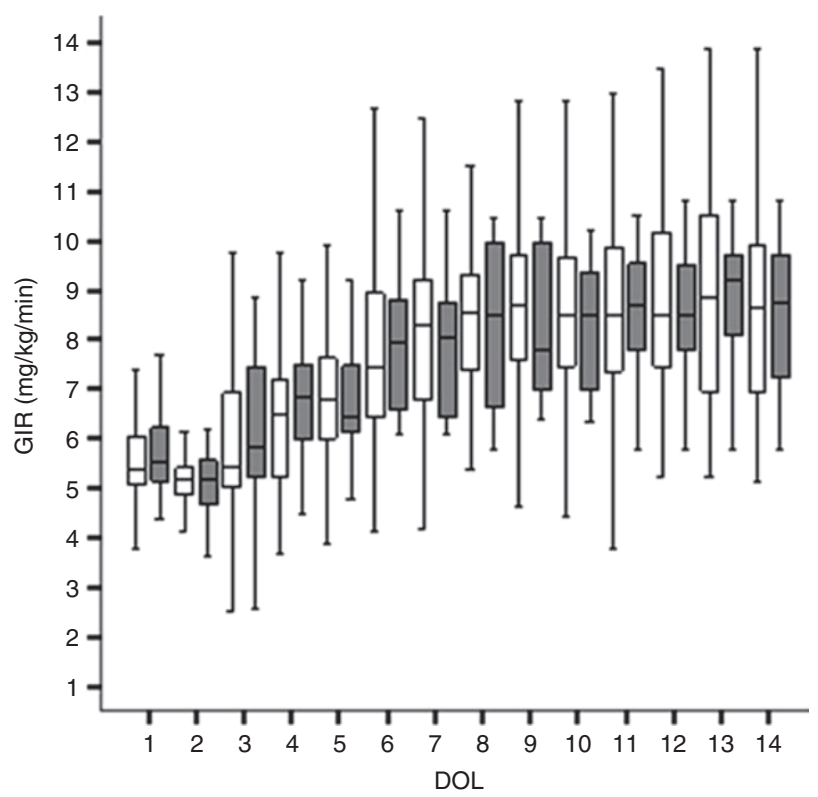

Figure 2. Glucose infusion rates (GIRs) by group. Daily median GIRs are expressed as $\mathrm{mg} / \mathrm{kg} / \mathrm{min}$ and compared for each day of life, with interquartile ranges shown as black lines. The nonsevere HG group is shown in white boxes and the severe HG group in dark boxes. DOL, day of life; HG, hyperglycemia.

mean serum glucose level was $159 \pm 69 \mathrm{mg} / \mathrm{dl}$ for the severe HG group and $130 \pm 48 \mathrm{mg} / \mathrm{dl}$ for the nonsevere HG group $(P<0.001$, Table 1$)$. The severe HG group had significantly higher mean and maximum serum glucose levels along with wide variations of glucose (as expressed by glucose ranges, Table 1) as compared with the nonsevere HG group during the first 3 and $7 \mathrm{~d}$ of life $(P<0.01$ for mean, maximum, and range differences between groups, Table 1 ). Of note, the mean glucose infusion rate during these time periods was not different 

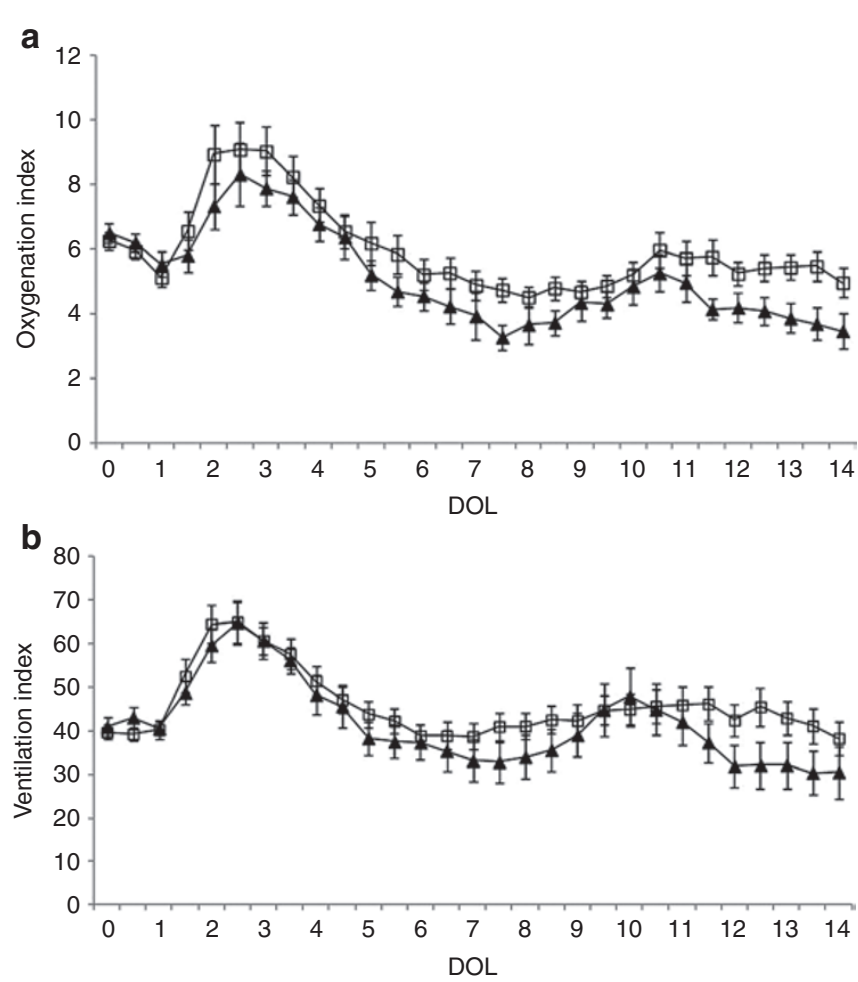

Figure 3. Daily oxygenation and ventilation indexes. (a) Daily oxygenation index and (b) ventilation index are shown as mean per day of life (DOL) in each group; SEs are shown. Empty squares represent the nonsevere HG group and black triangles represent the severe HG group (not significant). HG, hyperglycemia.

Table 2. Causes of early death

\begin{tabular}{ll}
\hline Severe hyperglycemia & Cause of death \\
\hline $\mathrm{Y}$ & Cardiac insufficiency $^{\mathrm{a}}$ \\
$\mathrm{Y}$ & Cardiac insufficiency $^{\mathrm{a}}$ \\
$\mathrm{Y}$ & Sepsis \\
$\mathrm{Y}$ & Renal insufficiency \\
$\mathrm{Y}$ & Cardiac insufficiency \\
$\mathrm{Y}$ & ${ }^{\mathrm{a}}$ \\
$\mathrm{N}$ & Cardiac insufficiency \\
$\mathrm{N}$ & Metabolic acidosis \\
$\mathrm{N}$ & Renal insufficiency \\
$\mathrm{N}$ & Unknown \\
$\mathrm{N}$ & Loss of tracheostomy \\
\hline $\mathrm{N}$, no; Y, yes. & Sepsis \\
${ }^{a}$ Cardiac insufficiency was defined as severe hypotension unresponsive to vasopressors \\
(dopamine, dobutamine, and epinephrine) and fluid resuscitation.
\end{tabular}

between the two groups (Figure 2). As expected, the numbers of hyperglycemic episodes in the first 3 and $7 \mathrm{~d}$ were higher in the severe HG group as compared with the nonsevere HG group (Table 1). There was no difference in the number of glucose measurements obtained during the first 3 and $7 \mathrm{~d}$ in the severe HG group as compared with the nonsevere group (15.2 \pm 4.1 vs. $14.7 \pm 3.9, P=0.6$, and $20.8 \pm 6.4$ vs. $23.2 \pm 4.3, P=$ 0.2 , respectively). The glucose ratios were higher at both 3 and $7 \mathrm{~d}$ in the severe HG group vs. the nonsevere HG group (0.55

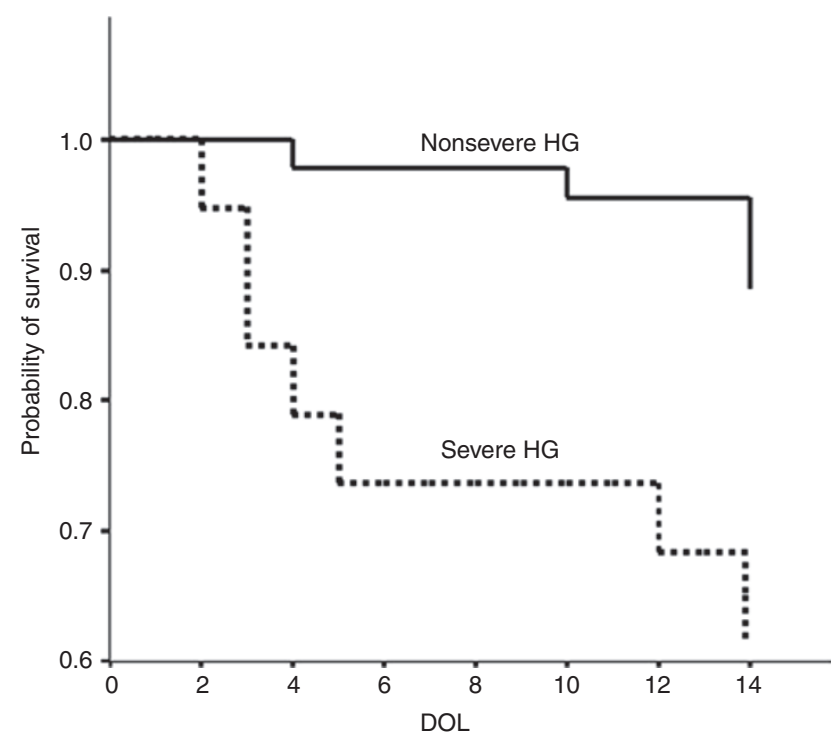

Figure 4. Probability of survival according to hyperglycemic status. This graph shows Kaplan-Meier survival curves comparing the nonsevere HG group (solid line) with the severe HG group (dashed line) $(P=0.05)$. HG, hyperglycemia.

\pm 0.12 vs. $0.32 \pm 0.18, P<0.001$, and $0.55 \pm 0.07$ vs. $0.28 \pm$ $0.11, P<0.001$, respectively). Urine glucose was measured a total of 1,011 times for all animals. Urine glucose was negative $(<100 \mathrm{mg} / \mathrm{dl})$ in $85 \%$ of the samples with a paired median serum glucose of $107 \mathrm{mg} / \mathrm{dl}$ (interquartile range $87-136$ ); $7.3 \%$ of the samples had trace glucose in urine $(\geq 100 \mathrm{but}<250 \mathrm{mg} / \mathrm{dl})$ with paired median serum glucose of $139 \mathrm{mg} / \mathrm{dl}$ (interquartile range 109-186), and $7.7 \%$ of the samples had $\geq 250 \mathrm{mg} / \mathrm{dl}$ of glucose in the urine with a paired median serum glucose of $195 \mathrm{mg} / \mathrm{dl}$ (interquartile range 155-265).

Insulin was used to treat $\mathrm{HG}$ in $70 \%$ of the animals (mean 1.7 $\pm 1.8 \mathrm{~d}$ of life). As expected, baboons with severe HG received more insulin doses than those with nonsevere HG (Table 1).

Cumulative enteral feed volume and the average amount of feeds per day were similar between the severe and nonsevere HG groups $(P=0.25)$. Because serum sodium can affect serum glucose, recorded daily measurements were compared, with no differences found between the two groups. A statistically significant difference in serum potassium levels was found between the severe and nonsevere $\mathrm{HG}$ groups at $3 \mathrm{~d}$ of life, probably due to the large number of measurements $(2,652$ and 5,337 measurements in the first 3 and $7 \mathrm{~d}$ of life, respectively) (Table 1); however, these differences were not clinically relevant.

\section{Clinical Outcomes}

Because animals were planned for euthanasia at day 14, the clinical outcome measures were early death and oxygenation and ventilation indexes. There were no differences in the daily oxygenation and ventilation indexes between the severe HG group and the nonsevere HG animals by independent $t$-test group comparisons at days 3 and 7 of life (Table 1), by binary logistic regression (data not shown), or by two-way ANOVA (Figure 3). Serum glucose levels did not correlate with 


\section{Articles | Blancoetal.}

oxygenation index or ventilation index $\left(r^{2}=0.001, r^{2}=0.001\right.$, respectively). Hemoglobin plays an important role in oxygenation and therefore, anemia was monitored. At day 3 of life, the severe HG group had a statistically lower hematocrit than the nonsevere HG group, but this level was considered to be clinically irrelevant and was probably due to the large number of measurements performed in each animal (Table 1). By $7 \mathrm{~d}$ of life, however, the hematocrit levels were similar between the two groups.

Early death occurred in 11 of 66 animals at a mean age of 6.7 $\pm 5 \mathrm{~d}$. One of the animals that did not die early was planned for experiment completion at $6 \mathrm{~d}$ instead of $14 \mathrm{~d}$; this animal was excluded from the analysis. Clinical characteristics in the early death group (weight, gender, oxygenation index, or ventilation index) did not differ from those of the rest of the animals (not significant). Severe HG was associated with early death, even after controlling for sepsis, postnatal steroid exposure, and catecholamine use (odds ratio 3.88, 95\% confidence interval 1.0214.81). As expected, the animals in the severe HG group had a shorter length of stay (secondary to increased number of early deaths) as compared with the nonsevere group, but due to sample size, it did not reach statistical significance (Table 1). The numbers of early deaths per quartile of mean plasma glucose levels were as follows: low quartile $(n=4)$; second and third quartile $(n=1)$; and high quartile $(n=6)$. When severe HG was categorized by tertiles or by highest glucose alone, the association with death did not reach statistical significance (data not shown). The cause of early death for each animal is shown in Table 2. One animal died due to loss of tracheostomy, which is unlikely to be associated to HG. This animal was not taken out of the analysis because it was in the nonsevere HG group and therefore, will unlikely contribute to any difference to the outcome measure (death). The probability of survival was lower in the severe HG group but did not reach statistical significance (Figure 4, $P=0.05$ ).

\section{DISCUSSION}

HG is a known condition that frequently occurs in preterm infants. The development of HG has been associated with significant morbidity in these infants, leading to long-term consequences and increased hospital stay $(1,3,4,6,7,16)$. Increased mortality has been well documented in diabetic adults, nondiabetic elderly patients, and critically ill children with poor glucose control (17-19); available data support increased mortality in preterm infants who develop HG early in life $(2,8)$.

In this study of 66 extremely preterm baboons, a majority of animals were found to be hyperglycemic during the first week of life, and prolonged exposure (as occurred in $29 \%$ of these animals) was associated with early death. Recent studies suggest a link between severe HG and increased odds of dying within the first week of life in extremely low birth weight (ELBW) preterm infants (8). Although this increased mortality may reflect severity of illness rather than causality, the odds of early death remained high even when controlling for other variables that express severity of illness, such as sepsis, postnatal steroid exposure, and catecholamine utilization. In fact, both groups of hyperglycemic animals (severe and nonsevere) had very similar clinical and demographic characteristics. Pulmonary functions, expressed by oxygenation and ventilation indexes, were followed very closely and were found to be similar between the two groups (Table 1 and Figure 3). Nonetheless, the pulmonary function in the severe HG group (Figure 3 ) appeared to be slightly better than that in the nonsevere HG group in the last $4 \mathrm{~d}$ of life (not statistically significant); this is probably due to lack of data from those animals that died early, skewing the data from healthier survivors. Nutritional management was dictated by parenteral nutrition and enteral feeding protocols set $a$ priori and no differences were found (Table 1). Glucose infusion rates were also similar between the two groups during the first week of life (Table 1 and Figure 2); therefore, the development of severe HG was unlikely to be due to differences in glucose administration. Another strength of this study relies on the frequent glucose measurements obtained per animal (more than 3,000 measurements in all), which is contrary to most clinical studies in which sick infants account for the majority reported to undergo various tests; the number of glucose measurements did not differ between groups because the protocol was strictly followed (as shown in the Results section). Although animals with $\mathrm{HG}>200 \mathrm{mg} / \mathrm{dl}$ received spot doses of insulin and may have had more glucose measurements, there were no differences in the number of measurements between groups, probably due to the high incidence of HG. This potential bias is further minimized by utilizing a glucose ratio to assess the time spent in a hyperglycemic state, which is a result of the number of times the animal was hyperglycemic divided by the number of times glucose was measured, rather than single hyperglycemic events.

The majority of animals (67\%) required vasoactive medications due to their severity of illness from extreme prematurity, but no differences were found in the number of cumulative amounts per day of catecholamine administration between the severe HG and nonsevere HG groups at different time points (Table 1). Due to the effect of vasoactive medications in gluconeogenesis (20), the use of these medications may have contributed to the high serum glucose levels and the incidence of HG. Most hyperglycemic events occurred within the first $72 \mathrm{~h}$ of life (Table 1) and the glucose levels measured were often $>150 \mathrm{mg} / \mathrm{dl}$, particularly within the first $2 \mathrm{~d}$ of life (Figure 1). Vasoactive medications were initiated at a mean of $37 \pm 36 \mathrm{~h}$ of life and may have contributed to prolonging the duration of HG. Nevertheless, the amount of vasoactive medications utilized was the same between groups. We may further speculate that the high incidence of $\mathrm{HG}$ in the first $2 \mathrm{~d}$ of life may have led to hyperosmolar dehydration, which in turn may have placed all animals at higher risk of requiring vasoactive support and those with persistent $\mathrm{HG}$ at an increased risk of death due to hypovolemia. Furthermore, glucosuria was initially found with median serum glucose of $139 \mathrm{mg} / \mathrm{dl}$ and increased with worsening HG. Finally, most of the deaths were due to unexplained renal/cardiac insufficiency and metabolic acidosis, which may be a consequence of hypovolemia due to hyperglycemic dehydration.

As evidenced in diabetic patients, it is likely that persistent exposure to HG will lead to greater consequences, whereas variations in glucose time/length of exposure may lead to 
differences in type of morbidity and mortality. For example, wide variations, the maximum serum glucose level reached, and the percentage of time spent in the hyperglycemic state (glucose ratio) may contribute to disease processes due to changes in osmolality, fluid shifts, and direct cellular effects in various organs, particularly in the fragile preterm brain (2). Among our study cohort, the severe HG group had higher mean glucose levels, wider fluctuations in glucose (expressed as max-min in Table 1), and higher peak levels during the first $3 \mathrm{~d}$ of life as compared with the nonsevere HG group $(P<0.01$ for mean, maximum, and max-min of serum glucose between groups, Table 1). These independent group differences persisted for $7 \mathrm{~d}$ of life and may have further contributed to the higher mortality rates, but due to sample size, the effect of each type of glucose exposure or contribution from other variables could not be determined.

Previous studies have suggested a relationship between sepsis and the development of HG; this was not observed in this study. This lack of association may be secondary to the fewer number of study animals that had culture-proven sepsis $(4.5 \%)$. The incidence of sepsis in ELBW infants is much higher $(21 \%$, from National Institute of Child Health and Human Development data) (21); this difference may be due to low risk of early sepsis secondary to the lack of maternal chorioamnionitis in planned preterm delivery by cesarean section in healthy animals and selection bias because animals did not live long enough to develop the disease (alive for $14 \mathrm{~d}$ ).

A weakness of the study might be the high incidence of $\mathrm{HG}$ (98\%). Although this incidence is higher than what has been reported in ELBW infants $(3,16,22)$, it is only $10-20 \%$ higher. In addition, most human studies are from nonhomogeneous ELBW populations. This population of animals is homogeneous, and the gestational age is very narrow $( \pm 2 \mathrm{~d})$. Therefore, the results of this study may be more representative of an ELBW infant with no confounding perinatal stress factors. This in turn may inflate the incidence of $\mathrm{HG}$ as a result of a lack of maturational effects in glucose metabolism from stress (i.e., cortisol, thyroid, and/or catecholamine surges). Another potential pitfall of this study is that causes of death may be more aggressively treated in human infants, but due to the great economic demands and involvement of highly skilled professionals in the non-human primate preterm model, a significant attempt to avoid death was sought at all times, which is less common in other animal models. Death alone as an outcome measure may be a limitation of this study given that it was planned at $14 \mathrm{~d}$ of age, and additional spontaneous deaths might have occurred afterward. HG commonly occurs in the first $2 \mathrm{wk}$ of life $(3,6)$; therefore, it is likely that we have captured the majority of early deaths that would have occurred as a consequence of HG, but we may have missed those resulting from late-onset $\mathrm{HG}$ or those secondary to longterm effects of this condition.

HG is a common side effect of postnatal dexamethasone therapy in preterm infants (23). In our study, only $30 \%$ of the hyperglycemic animals were exposed to postnatal steroids (hydrocortisone), and exposures to steroids were similar between groups (Table 1).
The pathogenesis of $\mathrm{HG}$ in critically ill preterm infants seems to be multifactorial, including abnormalities probably related to prematurity (persistent gluconeogenesis, inadequate pancreatic insulin release, and underdeveloped insulin-signaling pathways in the muscle $(5,9-11)$ ), in addition to superimposed postnatal factors (sepsis, medications). Although it is well known that stress and severity of illness can contribute to development of HG, this was not evident in our study population. This is a homogeneous population of extremely ill, preterm animals that received prenatal steroids. This population is very difficult to obtain given that there are multiple genetic and environmental differences in human populations. Therefore, this is a highly suitable model for studying the effects of HG.

Among critically ill adults, better glucose control improves clinical outcomes (24), but intensive insulin therapy leads to a higher incidence of death (25). For critically ill children with cardiac surgery, trauma, sepsis, or burns, HG has been shown to correlate with worse outcomes (26-29). Studies in newborns are limited, and so far, insulin therapy does not seem to decrease the incidence of HG, but tight glucose control has not been evaluated (30). Others have attempted early insulin therapy in very low birth weight preterm infants (without $\mathrm{HG}$ ), but this strategy was linked to higher mortality at $28 \mathrm{~d}$ of life; it is important to note that in some of these studies, the majority of infants were normoglycemic when insulin infusions were initiated (31). As expected, baboons in the current study with severe HG received more insulin doses than those with nonsevere HG. Insulin is more commonly given as a continuous infusion in human preterm infants (31), and insulin boluses may have contributed to a wider range of serum glucose seen in the severe HG group. One may argue that this may have contributed to the higher mortality rate seen in the severe hyperglycemic group, but it is unlikely that a difference of two extra doses of insulin in the severe HG group was clinically relevant as a cause of mortality. On the other hand, the majority of deaths occurred in the low (four deaths) and high (six deaths) mean plasma glucose quartiles. These findings may reflect the condition seen in diabetic adults, in which patients with low glucose have an increased risk of death (25).

Additional studies need to be performed to determine the highest level of blood glucose that safely provides the maximum amount of nutrients without increasing the risk of morbidity/ death. Moreover, it is well documented that preterm and smallfor-gestational-age infants are at an increased risk of cardiovascular disease and diabetes early in life; therefore, the long-term consequences of exposure to high glucose levels at critical periods of development need to be evaluated (32-34). It has been demonstrated that maternal HG leads to altered embryogenesis secondary to apoptotic effects, altered mitochondrial physiology, and generation of oxygen radicals resulting in neural tube, musculoskeletal, and cardiac defects $(35,36)$; in addition, exposure to gestational diabetes is associated with an increased incidence of adolescent obesity and glucose intolerance in the offspring. Therefore, exposures to high glucose levels during the extrauterine life at critical periods of development may have lifelong consequences (33). 
The current study demonstrates that the preterm baboon is a novel and clinically relevant model for the study of neonatal HG. Furthermore, preterm baboons develop conditions in the neonatal period that are similar to those in human infants (11-13). Previously, it has been demonstrated that the adult baboon is a pertinent nonhuman primate model to examine mechanisms affecting aberrant glucose control; baboons have close (97\%) phylogenetic proximity with humans and can develop insulin resistance and diabetes spontaneously (14). Therefore, the results of this study are highly translational to humans.

In conclusion, extremely preterm baboons have a high incidence of HG and severe HG occurring during the first week of life, which is associated with early death. No other morbidities were associated with severe HG. Future studies of this model will enhance our understanding of an optimal glycemic management to ameliorate morbidity and mortality in premature infants.

\section{MATERIALS AND METHODS \\ Animal Care}

A total of 66 preterm baboons were delivered at $67 \%$ of gestation at the Texas Biomedical Research Institute in San Antonio, Texas, from 2004 to 2008. Animals were delivered prematurely via cesarean section under general anesthesia from healthy, non-diabetic mothers at $125 \pm 2 \mathrm{~d}$ gestational age (full term $=185 \mathrm{~d}$ gestational age). All studies were approved by the institutional animal care committee at the Texas Biomedical Research Institute. Animal experiments were conducted in accordance with accepted standards of humane animal care. Mothers were given prenatal steroids initiated $48 \mathrm{~h}$ before delivery with betamethasone (6 mg intramuscular) every $24 \mathrm{~h}$ for two doses or dexamethasone (if there was a pharmaceutical shortage of betamethasone). This dose of prenatal steroids is equivalent to the dose used in humans when averaged as $\mathrm{mg} / \mathrm{kg}$ of body weight.

The animals were intubated immediately after birth and chronically ventilated for a planned survival of $14 \mathrm{~d}$, except for one animal in which survival was only planned for $6 \mathrm{~d}$. None of the animals included in this study received any investigational treatments. Surfactant (Survanta, Abbott Laboratories, Abbott Park, IL) was administered through the endotracheal tube immediately after placement at a dose of $4 \mathrm{ml} / \mathrm{kg}$. A standard protocol was followed for sedation and anesthesia allowing animals movement similar to what is tolerated in preterm ventilated infants. This was achieved by titrating the animals' sedation as needed at an approximate interval of every $2-4 \mathrm{~h}$. A detailed protocol for ventilator management was followed according to blood gas analysis; blood gases were measured hourly for the first $48 \mathrm{~h}$ and then every $2 \mathrm{~h}$ for the rest of the experiment. Central intravenous lines were placed shortly after birth for fluid management and parenteral nutrition. An echocardiogram was obtained on the first day of life and daily thereafter; details have been published elsewhere $(13,37)$. A $5 \%$ dextrose intravenous solution was started after birth at a rate of $150 \mathrm{ml} / \mathrm{kg} / \mathrm{d}$. The glucose infusion rate was increased every $2 \mathrm{~d}$ per protocol (by $1-2 \mathrm{mg} / \mathrm{kg} / \mathrm{min}$ ), and total fluid goal was titrated depending on fluid balance. Measurements of glucose levels were obtained shortly after birth and then at a minimum of every $6 \mathrm{~h}$ using the AU 640 Immuno Analyzer (Olympus, San Diego, CA). Additional glucose measurements were obtained if the animals were found to be hyperglycemic. Three professionals were in charge of the daily management (two neonatologists/one neonatal nurse practitioner); there could be requests for additional measurements but not fewer. The direct caregivers (technicians) were very familiar with the protocols and consistently followed procedures. Urine glucose was measured at least every $12 \mathrm{~h}$ by Multistix 10 SG reagent strips for urinalysis (Siemens (Bayer) Medical Solutions, Pittsburg, PA). Intravenous insulin was given as a bolus administration dose of $0.5 \mathrm{IU} / \mathrm{kg}$ to treat serum glucose levels $>200 \mathrm{mg} / \mathrm{dl}$. After insulin administration, glucose measurements were monitored at a minimum interval of $4 \mathrm{~h}$ until euglycemia was achieved. A parenteral feeding protocol was initiated after $24 \mathrm{~h}$ of life. Intravenous amino acids were started at $24 \mathrm{~h}$ of life at a dose of $1.75 \mathrm{~g} / \mathrm{kg}$, increased to
$3.5 \mathrm{~g} / \mathrm{kg}$ by $48 \mathrm{~h}$ of life, and maintained at that dose for the remainder of the days in the protocol. Intravenous lipids were started at $24 \mathrm{~h}$ of life at a dose of $1 \mathrm{~g} / \mathrm{kg}$ and increased incrementally to a maximum of $3 \mathrm{~g} / \mathrm{kg} /$ day. Enteral feeds were initiated at $3 \mathrm{~d}$ of age, if bowel gas pattern was considered normal on radiograph. Primalac formula (BioServ, Frenchtown, $\mathrm{NJ}$ ) was initiated as trophic feeds and increased as tolerated by $20 \mathrm{ml} / \mathrm{kg} /$ day to a maximum feeding volume of $150 \mathrm{ml} / \mathrm{kg} /$ day. Dopamine, dobutamine, epinephrine, and hydrocortisone were initiated in that order and advanced to a set maximum amount to maintain mean arterial pressure $>25 \mathrm{~mm} \mathrm{Hg}$. A weaning protocol was followed as well to avoid hypertension. Sepsis was defined by at least one positive blood, urine, or cerebrospinal fluid culture. Prophylactic antibiotics were administered for $48 \mathrm{~h}$ to mimic human care. Thereafter, antibiotics were continued or restarted based on clinical condition.

\section{Data Collection}

Demographic data, including birth weight, gender, length of stay, and death, were recorded in the medical chart. Clinical data collected included all serum glucose measurement and chemistries, medications (catecholamines (dopamine, dobutamine, epinephrine), insulin, and steroids), glucose infusion rate (calculated hourly throughout stay), hourly ventilator settings, urinalysis, sepsis (defined as a positive blood, urine, or cerebrospinal fluid culture), and any other pertinent clinical diagnosis.

Oxygenation index was calculated as $\left(\mathrm{MAP} \times \mathrm{FiO}_{2}\right) / \mathrm{pO} \times 100$, where $\mathrm{MAP}$ is the mean airway pressure, $\mathrm{FiO}_{2}$, is the fraction of inspired oxygen, and $\mathrm{pO}_{2}$, is the partial pressure of oxygen. Ventilation index was calculated as $\left(\mathrm{PIP} \times\right.$ respiratory rate $\left.\times \mathrm{pCO}_{2}\right) / 1,000$, where PIP is the peak inspiratory pressure and $\mathrm{pCO}_{2}$, is the partial pressure of carbon dioxide. The oxygenation and ventilation indexes were recorded every $2-4 \mathrm{~h}$, but for statistical purposes, an average was calculated at 12-h intervals for each animal. Due to the higher mortality in the severe HG group, the data sets for the two groups became unbalanced and therefore, an ANOVA for repeated measures could not be accomplished. Consequently, a twoway ANOVA was done to compare the oxygenation index and ventilation index with glucose level and time as the two parameters.

HG was defined as a serum glucose concentration of $\geq 150 \mathrm{mg} / \mathrm{dl}$ $(8.3 \mathrm{mmol} / \mathrm{l})$ during the first $7 \mathrm{~d}$ of life. This level has been previously identified as being clinically relevant in the human population $(38,39)$. Fasting serum glucose levels in preterm baboons, term baboons, and adult baboons were $40.6 \pm 4.7,59.2 \pm 10.6$, and $87.0 \pm 16.6 \mathrm{mg} / \mathrm{dl}$, respectively (40). Therefore, HG as defined earlier is highly relevant in the baboon model.

For logistic regression analyses, the animals were defined according to their top quartile distribution for severity of HG. The top quartile (i.e., severe $\mathrm{HG}$ group) was defined by having $\geq 8$ episodes (glucose measurement $\geq 150 \mathrm{mg} / \mathrm{dl}$ ) of HG during the first $7 \mathrm{~d}$ of life. In addition, to ensure that this group had in fact a greater exposure to $\mathrm{HG}$, and not only a greater number of measurements, a glucose ratio was calculated for all animals. This was defined as the number of hyperglycemic events divided by the number of total measurements. A glucose ratio has been previously found to be a good marker of the time spent in hyperglycemic state and may reflect length of exposure (6). The glucose ratio utilized by Hays et al. was different because in humans the number of measurements per day was not standardized, and a time ratio for glucose concentrations of $>150 \mathrm{mg} / \mathrm{dl}$ was calculated as the number of days with a lowest blood glucose value of $>150 \mathrm{mg}$, divided by the total number of days on which blood glucose concentrations were obtained during the first week of life. In this study, because measurements were standardized by a protocol with a minimum of four measurements per day, and the incidence of $\mathrm{HG}$ was so high, we used a glucose ratio to ensure that the animals with "severe" HG were not classified as such because glucose was checked more frequently but rather because they spent more time hyperglycemic.

Serum glucose fluctuation was calculated daily by deducting the minimum level from the maximum level in each animal. Similarly, minimum and maximum serum glucose levels were recorded daily.

\section{Statistical Methods}

Statistical calculations were performed with SPSS for Windows (Version 16.5; SPSS, Chicago, IL) and Prism (version 4.0; GraphPad Software, La 
Jolla, CA). Distributions and means of demographic and clinical variables were compared across study groups using $\chi^{2}$ tests and $t$-tests, and the Mann-Whitney test was used when data were not normally distributed. SDs are shown for continuous variables when means are calculated, and interquartile ranges are shown for variables when medians are calculated. Binary logistic regression analysis was used to assess correlates of $\mathrm{HG}$ and to assess the association of $\mathrm{HG}$ with several clinical outcomes, after adjusting for birth weight, catecholamine exposure, postnatal steroid use, presence of sepsis, and/or occurrence of early death when appropriate. Kaplan-Meier curves were used to assess probability of survival, and Pearson correlations were used with selected continuous variables. Two-way ANOVA and repeated measures were used to identify within- and between-subject effects of continuous variables.

\section{ACKNOWLEDGMENTS}

We thank Steve Seidner and Ralph DeFronzo for their advice and continual support. We also thank the personnel at the University of Texas Health Science Center and Texas Biomedical Research Institute for their dedication to this project.

\section{STATEMENT OF FINANCIAL SUPPORT}

This study was supported by grants from the Robert Wood Johnson Foundation (grant 67067 to C.L.B.), Clinical and Translational Science Award (grant UL1RR025767 to C.L.B.), American Diabetes Association (grant 7-11-BS-13 to C.L.B.), and the National Institutes of Health (grant HL52636 to the Bronchopulmonary Dysplasia Resource Center and National Center for Research Resources grant P51 RR013986 to the Texas Biomedical Research Institute).

\section{REFERENCES}

1. Garg R, Agthe AG, Donohue PK, Lehmann CU. Hyperglycemia and retinopathy of prematurity in very low birth weight infants. J Perinatol 2003;23:186-94.

2. Alexandrou G, Skiöld B, Karlén J, et al. Early hyperglycemia is a risk factor for death and white matter reduction in preterm infants. Pediatrics 2010;125:e584-91.

3. Blanco CL, Baillargeon JG, Morrison RL, Gong AK. Hyperglycemia in extremely low birth weight infants in a predominantly Hispanic population and related morbidities. J Perinatol 2006;26:737-41.

4. Hall NJ, Peters M, Eaton S, Pierro A. Hyperglycemia is associated with increased morbidity and mortality rates in neonates with necrotizing enterocolitis. J Pediatr Surg 2004;39:898-901; discussion 898-901.

5. Liechty EA. The resistant premie: documenting the prevalence of hyperglycemia in the extremely low birth weight infant. J Pediatr 2010;157:699-700.

6. Hays SP, Smith EO, Sunehag AL. Hyperglycemia is a risk factor for early death and morbidity in extremely low birth-weight infants. Pediatrics 2006;118:1811-8.

7. Kaempf JW, Kaempf AJ, Wu Y, Stawarz M, Niemeyer J, Grunkemeier G. Hyperglycemia, insulin and slower growth velocity may increase the risk of retinopathy of prematurity. J Perinatol 2011;31:251-7.

8. Kao LS, Morris BH, Lally KP, Stewart CD, Huseby V, Kennedy KA. Hyperglycemia and morbidity and mortality in extremely low birth weight infants. J Perinatol 2006;26:730-6.

9. Mitanchez-Mokhtari D, Lahlou N, Kieffer F, Magny JF, Roger M, Voyer $\mathrm{M}$. Both relative insulin resistance and defective islet beta-cell processing of proinsulin are responsible for transient hyperglycemia in extremely preterm infants. Pediatrics 2004;113(3 Pt 1):537-41.

10. Chacko SK, Ordonez J, Sauer PJ, Sunehag AL. Gluconeogenesis is not regulated by either glucose or insulin in extremely low birth weight infants receiving total parenteral nutrition. J Pediatr 2011;158:891-6.

11. Blanco CL, Liang H, Joya-Galeana J, DeFronzo RA, McCurnin D, Musi N. The ontogeny of insulin signaling in the preterm baboon model. Endocrinology 2010;151:1990-7.

12. Escobedo MB, Hilliard JL, Smith F, et al. A baboon model of bronchopulmonary dysplasia. I. Clinical features. Exp Mol Pathol 1982;37:323-34.

13. McCurnin D, Clyman RI. Effects of a patent ductus arteriosus on postprandial mesenteric perfusion in premature baboons. Pediatrics 2008;122:e1262-7.

14. Chavez AO, Lopez-Alvarenga JC, Tejero ME, et al. Physiological and molecular determinants of insulin action in the baboon. Diabetes 2008;57:899-908.

15. Guardado-Mendoza R, Dick EJ Jr, Jimenez-Ceja LM, et al. Spontaneous pathology of the baboon endocrine system. J Med Primatol 2009;38:383-9.
16. van der Lugt NM, Smits-Wintjens VE, van Zwieten PH, Walther FJ. Short and long term outcome of neonatal hyperglycemia in very preterm infants: a retrospective follow-up study. BMC Pediatr 2010;10:52.

17. Muggeo M, Verlato G, Bonora E, Zoppini G, Corbellini M, de Marco R. Long-term instability of fasting plasma glucose, a novel predictor of cardiovascular mortality in elderly patients with non-insulin-dependent diabetes mellitus: the Verona Diabetes Study. Circulation 1997;96:1750-4.

18. Muggeo M, Verlato G, Bonora E, et al. Long-term instability of fasting plasma glucose predicts mortality in elderly NIDDM patients: the Verona Diabetes Study. Diabetologia 1995;38:672-9.

19. Rake AJ, Srinivasan V, Nadkarni V, Kaptan R, Newth CJ. Glucose variability and survival in critically ill children: allostasis or harm? Pediatr Crit Care Med 2010;11:707-12.

20. Shiroyama K, Moriwaki K, Yuge O. The direct effect of dopamine on glucose release from primary cultured rat hepatocytes. In Vivo 1998;12:527-9.

21. Stoll BJ, Hansen N, Fanaroff AA, et al. Late-onset sepsis in very low birth weight neonates: the experience of the NICHD Neonatal Research Network. Pediatrics 2002;110(2 Pt 1):285-91.

22. Beardsall K, Vanhaesebrouck S, Ogilvy-Stuart AL, et al. Prevalence and determinants of hyperglycemia in very low birth weight infants: cohort analyses of the NIRTURE study. J Pediatr 2010;157:715-9.e1-3.

23. Halliday HL, Ehrenkranz RA, Doyle LW. Early ( $<8$ days) postnatal corticosteroids for preventing chronic lung disease in preterm infants. Cochrane Database Syst Rev 2010;1:CD001146.

24. Van den Berghe G, Wilmer A, Hermans G, et al. Intensive insulin therapy in the medical ICU. N Engl J Med 2006;354:449-61.

25. Finfer S, Chittock DR, Su SY, et al. Intensive versus conventional glucose control in critically ill patients. N Engl J Med 2009; 360:1283-97.

26. Preissig CM, Rigby MR, Maher KO. Glycemic control for postoperative pediatric cardiac patients. Pediatr Cardiol 2009;30:1098-104.

27. Garcia Branco R, Tasker RC, Ramos Garcia PC, Piva JP, Dias Xavier L. Glycemic control and insulin therapy in sepsis and critical illness. J Pediatr (Rio J) 2007;83:Suppl 5:S128-36.

28. Bochicchio GV, Sung J, Joshi M, et al. Persistent hyperglycemia is predictive of outcome in critically ill trauma patients. J Trauma 2005;58:921-4.

29. Gore DC, Chinkes D, Heggers J, Herndon DN, Wolf SE, Desai M. Association of hyperglycemia with increased mortality after severe burn injury. J Trauma 2001;51:540-4.

30. Ng SM, May JE, Emmerson AJ. Continuous insulin infusion in hyperglycaemic extremely-low- birth-weight neonates. Biol Neonate 2005;87:26972.

31. Beardsall K, Dunger D. Insulin therapy in preterm newborns. Early Hum Dev 2008;84:839-42.

32. Whincup PH, Kaye SJ, Owen CG, et al. Birth weight and risk of type 2 diabetes: a systematic review. JAMA 2008;300:2886-97.

33. Catalano PM, Kirwan JP, Haugel-de Mouzon S, King J. Gestational diabetes and insulin resistance: role in short- and long-term implications for mother and fetus. J Nutr 2003;133:5 Suppl 2:1674S-83S.

34. Hofman PL, Regan F, Jackson WE, et al. Premature birth and later insulin resistance. N Engl J Med 2004;351:2179-86.

35. Chi MM, Hoehn A, Moley KH. Metabolic changes in the glucose-induced apoptotic blastocyst suggest alterations in mitochondrial physiology. Am J Physiol Endocrinol Metab 2002;283:E226-32.

36. Wang R, Martínez-Frías ML, Graham JM Jr. Infants of diabetic mothers are at increased risk for the oculo-auriculo-vertebral sequence: a case-based and case-control approach. J Pediatr 2002;141:611-7.

37. McCurnin DC, Yoder BA, Coalson J, et al. Effect of ductus ligation on cardiopulmonary function in premature baboons. Am J Respir Crit Care Med 2005;172:1569-74.

38. Cowett RM, Farrag HM. Selected principles of perinatal-neonatal glucose metabolism. Semin Neonatol 2004;9:37-47.

39. Denne SC, Poindexter BB, Leitch CA, Ernst JA, Lemons PK, Lemons JA. Nutrition and metabolism in the high risk neonate. In: Fanaroff AA, Martin RJ, eds. Neonatal-Perinatal Medicine. Diseases of the Fetus and Infant. Philadelphia, PA: Mosby-Elsevier, 2006:661-91.

40. Quinn AR, Blanco CL, Perego C, et al. The ontogeny of the endocrine pancreas in the fetal/newborn baboon. J Endocrinol 2012;214:289-99. 